\title{
Mapping of Rescue Environment Based on NDT Scan Matching
}

\author{
Jinliang Li, Youxia Sun \\ School of Mechanical and Electronics Engineering \\ Shandong University of Science and Technology \\ Qingdao, 266590, China \\ E-mail: lixuanci@gmail.com
}

\begin{abstract}
This paper studied the mapping problem for rescue robots based on laser scan matching and extend Kalman filtering (EKF). Because of the non-structural rescue environments, it is hard to extract typical features. Scan matching method based on normal distribution transform (NDT) can avoid the hard feature extraction problem by estimation of the probability distribution of laser scan data. By fusing NDT scan matching with EKF framework, the NDTEKF SLAM algorithm was proposed, which can effectively and precisely build maps for rescue environment. Experiment results show that NDT-EKF SLAM algorithm is more precise than algorithms based solely on scan-matching.
\end{abstract}

Keywords-Rescue robots; Scan matching; SLAM; Normal distribution transform

\section{INTRODUCTION}

For protecting humans, it is nowadays very important to build robots that are able to operate in earthquake, fire, explosive and chemical disaster areas [1]. During Rescue process, rescuers have limited time to find survivors (typically 10 hours) [2]. Under this emergent circumstance, rescue robots can help the rescuers. Recently, there has been a surge in the research on rescue robots. However until now, there have been no systems that can reliably navigate in rescue environment like mines [3]. To navigate reliably in unknown environment, the rescue robot must have the ability of simultaneous localization and mapping (SLAM) [4] [6]. The SLAM problem has been thoroughly researched theoretically and has been demonstrated many times on mobile robots in structured environment. The predominant form of SLAM to date is stochastic SLAM as introduced by Smith, Self and Cheeseman[5]. However in unstructured rescue environment little work has been done due to the difficulty in feature extraction and data association problem for conventional SLAM.

In [7] and [8], the authors proposed SLAM based on scan matching in unstructured environment to overcome the difficulties in feature extraction and data association. The dominant scan-matching method used in mobile robotics today was introduced by [11], a variant of ICP(Iterative Closet Points algorithm) applied to laser scan matching [10]. The ICP algorithm requires explicit point correspondence have to be established, which is a difficult task. Peter Biber proposed NDT (Normal Distribution Transform) as a compelling alternative for scan matching, which has been proved more efficient than ICP [12]. The NDT scan matching needs much less memory than ICP, which is very important for the large scale rescue environment.
This paper present a new approach to SLAM which is based on integration of NDT scan matching with the EKF SLAM frame work(we call it NDT-EKF SLAM) . We use laser data acquired with a 2D laser range finder, along with odometry. As the vehicle moves, we divide this data into 2D point clouds, each reference to a vehicle pose. This is achieved by using a relatively straightforward segmentation algorithm, avoiding entirely the need to periodically stop and take data. As vehicle poses with attached $2 \mathrm{D}$ point sets are formed, odometry provides dead-reckoned transformations between them. These are then fused in the EKF SLAM framework.

Using NDT-EKF SLAM has several distinct advantages. Firstly, NDT scan matching overcome difficulties in data association. Secondly, NDT-EKF SLAM can be deployed in large scale environment, which is critically important for real SLAM application. Thirdly, due to the probabilistic nature of NDT-EKF SLAM, the entire state can be updated with a single observation, when loop closure is detected.

The rest of this paper is structured as follows:

Section II begins by describing our segmentation and preprocessing method for laser scan data. NDT scan matching is described thoroughly in Section III, while Section VI covers the mathematics behind the Delayed State EKF SLAM. Section V contains experimental results and conclusions can be found in section VI.

\section{SCAN MATCHING BASED ON NDT}

\section{A. Preprocessing of Laser Scan Data}

Scanning is noisy and small errors may occur, namely Gaussian noise and salt pepper noise. The latter one arises for example at edges, where the laser beam of the scanner hits two surfaces, resulting in mean and erroneous data value. Furthermore reflections, e.g. at glass surfaces lead to suspicious data. We use filtering methods proposed in [9] to modify the data in order to enhance the quality of each scan, typically containing 181 data points. The data reduction, used for Gaussian noise, works as follows: the scanner emits the laser beams in a spherical way, such that the data points close to the source are denser. Multiple data points located close together are joined into one point. The number of these so-called reduced points is one order of magnitude smaller than the original one. For eliminating salt and pepper noise, a median filter removes the outliers by replacing a data point with the median value of the $n$ surrounding points (here: $n=$ 5). The neighbor points are determined according to their index within the scan, since the laser scanner provides with the data stored in a counter-clockwise direction. The median 
value is calculated with regard to the point of origin. In order to remove noisy data but leave the remaining scan points untouched, the filtering algorithm replaces a data point with the corresponding median value if and only if the Euclidean distance between both is larger than a fixed threshold.

\section{B. Normal Distribution Transform}

The goal of matching two range scans is to find the relative pose between the two positions, at which the scans were taken. The basis for most successful algorithms is the establishment of correspondence primitives of the two scans. Out of this, an error measure can be derived. Following the nomenclature of Besl and Mckay [10], the scan that serves as reference is called the model and the scan that is moved into alignment with the model is called the data scan. The key element in NDT scan matching is its representation of the model. Instead of using the individual points of the model, it is represented by a combination of normal distributions, describing the probability of finding a surface point at a certain position. Because of the points in the model are not used directly for matching, there is no need for the computationally expensive nearest-neighbor search that is done in the central loop of ICP. Storing the point sets themselves also requires much less memory. This is beneficial for all large maps, especially in rescue environment.

The first step of NDT algorithm is to subdivide the space occupied by the model into regularly sized cells. Then, for each cell $b$ that contains more than some minimum number of points, the mean vector $\mathbf{q}$ of the points in the cell and the covariance matrix $\Sigma$ are calculated as:

$$
\begin{aligned}
& \mathbf{q}=\frac{1}{n} \sum_{i=1}^{n} \mathbf{x}_{i} \\
& \boldsymbol{\Sigma}=\frac{1}{n-1} \sum_{i=1}^{n}\left(\mathbf{x}_{i}-\mathbf{q}\right)\left(\mathbf{x}_{i}-\mathbf{q}\right)^{t}
\end{aligned}
$$

where $\mathbf{x}_{i=1, \cdots, n}$ are the points contained in the cell.

The probability that there is a point at position $\mathbf{x}$ in cell b can then be modeled by the normal distribution $\mathcal{N}(\mathbf{q}, \mathbf{\Sigma})$. The probability density function(PDF) is formulated as:

$$
p(\mathbf{x})=\frac{1}{c} \exp \left(\frac{-(\mathbf{x}-\mathbf{q})^{t} \boldsymbol{\Sigma}(\mathbf{x}-\mathbf{q})}{2}\right)
$$

Where $\mathbf{q}$ and $\Sigma$ are the mean vector and covariance matrix for the cell that contains point $\mathrm{x}$, and $\mathrm{c}$ is $\mathrm{a}$ normalizing constant that can be set to one for practical purposes. Setting the limit for which cells are considered occupied to five points per cell is reasonable, in order to get a sensible covariance matrix. A 2D laser scan and its corresponding normal distributions are shown in Fig. 1.

The parameters to be optimized-i.e. the rotation and translation of the current pose estimate-can be encoded in a vector p . For NDT can matching, there are three transformation parameters to be optimized. Let $\mathbf{p}=\left(t_{x}, t_{y}, \phi\right)$ be the parameter vector, where $t_{x}$ and $t_{y}$ are the translation parameters and $\phi$ is the rotation angle. Using counter-clockwise rotation, the 2D transformation function is:

$$
T(\mathbf{p}, \mathbf{x})=\left(\begin{array}{cc}
\cos \phi & -\sin \phi \\
\sin \phi & \cos \phi
\end{array}\right) \mathbf{x}+\left(\begin{array}{l}
t_{x} \\
t_{y}
\end{array}\right)
$$
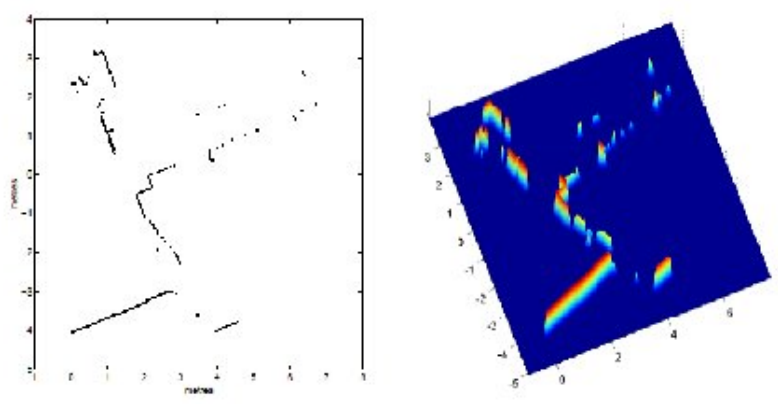

Fig 1. NDT of laser scan data

The algorithm measures the fitness of a particular pose by evaluation the PDFs at all points of the data scan. Since optimization problems are generally formulated as minimization problems, the score function is defined so that good parameters yield a large negative number.

Given a set of points $\mathcal{X}=\left\{\mathbf{x}_{1}, \cdots, \mathbf{x}_{n}\right\}$, a pose $\mathbf{p}$, and a transformation function $T(\mathbf{p}, \mathbf{x})$ to transform a point in the $2 \mathrm{D}$ plane, the score function $\operatorname{Score}(\mathbf{p})$ for the current set of parameters is defined as:

$$
\operatorname{Score}(\mathbf{p})=-\sum_{i=1}^{n} p\left(T\left(\mathbf{p}, \mathbf{x}_{i}\right)\right)
$$

In other words, the score is the negated sum of probabilities that the transformed points of the data scan are actually lying on the model curve. Given the transformation parameters $\mathbf{p}$, Newton's algorithm can be used to iteratively solve the equation $\mathbf{H} \Delta \mathbf{p}=-\mathbf{g}$, where $\mathbf{H}$ and $\mathbf{g}$ are the Hessian and gradient of Score $(\mathbf{p})$. The increment $\Delta \mathbf{p}$ is added to the current estimate of the parameters in each iteration, so that $\mathbf{p} \leftarrow \mathbf{p}+\Delta \mathbf{p}$. For more details, please refer to [12].

\section{CALCULATION OF COVARIANCE FOR SCAN MATCHING POSE}

To fuse pose acquired by scan matching and odometry data together, it is necessary to compute the covariance $\boldsymbol{\Sigma}$ of robot pose $\mathbf{x}_{v}=\left(x_{v}, y_{v}, \theta_{v}\right)^{t}$. Suppose $\mathbf{x}_{v}$ is Gaussian, then

$$
p\left(\mathbf{x}_{v}\right)=\mathcal{N}(\mu, \Sigma)
$$

Where $\mu$ is the mean of scan matching pose. The maximum value of $p\left(\mathbf{x}_{v}\right)$ is found at its mean $\mathbf{x}_{v}=\mu$. 
Denote it as $C_{m}$, note the equation (5) is sum of Gaussian ( SoG). To compute $\Sigma$, it is reasonable to approximate (5) by a single Gaussian in the vicinity of maximum value. For the a known robot pose $\mathbf{x}_{v i}$ by scan matching, we have:

$$
\begin{aligned}
c_{i}=\mathcal{N} & (\mu, \Sigma) \\
& =c_{m} \exp \left(\frac{-\left(\mathbf{x}_{v i}-\mu\right) \Sigma^{-1}\left(\mathbf{x}_{v i}-\mu\right)}{2}\right)
\end{aligned}
$$

By taking logs and rearranging terms, we get

$$
\left(\mathbf{x}_{v i}-\mu\right)^{t} \Sigma^{-1}\left(\mathbf{x}_{v i}-\mu\right)=-2\left(\ln c_{i}-\ln c_{m}\right)
$$

where $\mathbf{x}_{v i}, \mu, C_{i}, C_{m}$ are all known. So the inverse covariance matrix $\Sigma^{-1}$ (and hence $\Sigma$ ) can be evaluated. The result is an equation with six unknowns, and so a solution can be found given six samples from the Gaussian. This is posed as a matrix equation of the form:

$$
\mathbf{A x}=\mathbf{b}
$$

where the i-th row of $\mathbf{A}$ is $\left(x_{i}^{2}, 2 x_{i} y_{i}, 2 x_{i} \theta_{i}, y_{i}^{2}, 2 y_{i} \theta_{i}, \theta_{i}^{2}\right)$ and $\mathbf{x}$ is the unknown $(a, b, c, d, e, f)^{t}$, and $\mathbf{b}$ is the set of solutions. For a Gaussian function, the solution of this system of equations gives the exact covariance of the function. Since the scan correlation likelihood function is not exactly Gaussian (although we presume it has approximately Gaussian shape near the maximum likelihood location), different sets of samples will produce different values for $\boldsymbol{\Sigma}$. To reduce this variation, we evaluate more than the minimum number of samples and compute a least-squares solution using singular value decomposition (SVD), which results in a much more stable covariance estimate. The least-squares solution of (9) is:

$$
\mathbf{x}=\left(\mathbf{A}^{t} \mathbf{A}\right)^{-1} \mathbf{A}^{t} \mathbf{b}
$$

\section{NDT-EKF SLAM}

In contrast to conventional feature-based approaches, the world is represented by a series of past vehicle pose with associated uncertainties in NDT-EKF SLAM. Previous laser scan are 'attached' or associated to each pose. This is most commonly done immediately after odometry-based state augmentation, but is also essential when revisiting previously traversed areas for 'loop closing' [7].

\section{A. State Augmentation}

At time $\mathrm{t}=0$, the global state $\mathbf{x}$, only contains an initial vehicle pose, and the corresponding zero filled $3 \times 3$ covariance matrix $\mathbf{p}$. Given a noisy control input $\mathbf{u}(k+1)$ at time $\mathrm{k}+1$, the vehicle state evolves as shown:

$$
\mathbf{x}_{v(n+1)}(k+1 \mid k)=\mathbf{x}_{v n}(k \mid k) \oplus \mathbf{u}(k+1)
$$

where $\mathbf{x}_{v(n+1)}(k+1 \mid k)$ is the estimate of $\mathbf{x}_{v(n+1)}$, the $(\mathrm{n}+1)$ th vehicle pose at time $\mathrm{k}+1$ and the $\oplus$ operator signifies the composition operator, defined in [5]. If we also use the previous pose's covariance matrix $P_{v n}(k \mid k)$, and a control input noise covariance matrix $U$, the new pose's covariance matrix can be found as follows:

$$
\begin{aligned}
P_{v(n+1)}(k+1 \mid k) & =J_{1}\left(\mathbf{x}_{v n}, u\right) P_{v n}(k \mid k) J_{1}\left(\mathbf{x}_{v n}, u\right)^{t} \\
& +J_{2}\left(\mathbf{x}_{v n}, u\right) U J_{2}\left(\mathbf{x}_{v n}, u\right)^{t}
\end{aligned}
$$

where the $\mathrm{k}$ and $\mathrm{k}+1$ have been dropped from $\mathbf{x}_{v n}$ and $U$ for clarity, $J_{1}$ and $J_{2}$ are jacobians of the composition operator $\oplus$.

\section{B. State Update}

Let us assumed that an inter-pose scan matching has been triggered at time k between two scans $S_{i}$ and $s_{j}$, the first belong to pose $\mathbf{x}_{v}(i)$ from time i, the second belong to pose $\mathbf{x}_{v}(j)$ from time $\mathrm{j}$. This will yield an improved transformation estimated between the two pose.

Both poses are present in the global state vector $\mathbf{x}$, and therefore a predicted 'measurement' between the two poses can be found from the observation model as follows:

$$
\begin{aligned}
& T_{i, j}(k+1 \mid k)=h(\mathbf{x}(k+1) \mid k) \\
& =\ominus\left(\ominus \mathbf{x}_{v}(k+1 \mid k) \oplus \mathbf{x}_{v i}(k+1 \mid k)\right)
\end{aligned}
$$

where the $\Theta$ operator signifies the reverse transformation operator, as defined in [5], $\mathbf{x}_{v i}(k+1 \mid k)$ and $\mathbf{x}_{v j}(k+1 \mid k)$ refer to the $t=i$ and $t=j$ vehicle pose in $\mathbf{x}(k+1 \mid k)$ respectively. This is then used as the initial estimate for our scan matching algorithm as follows(as well as the predicted measurement in the update equations below):

$$
T_{i, j}(k+1)=\Psi\left(T_{i, j}(k+1 \mid k), S_{i}, S_{j}\right)
$$

where $\Psi$ represents our scan matching algorithm, detailed in section IV. The state update equations are then identical to the conventional EKF update equations. The innovation $\mathrm{V}$ and corresponding covariance $S$ can be found as follows:

$$
\begin{aligned}
& v=T_{i, j}(k+1)-T_{i, j}(k+1 \mid k) \\
& S=\nabla H_{x} P(k+1 \mid k) \nabla H_{x}^{t}+\Sigma
\end{aligned}
$$

where $\Sigma$ is the scan matching covariance matrix and $\nabla H$ is the jacobian of the observation model .

\section{EXPERIMENTAL RESULTS}

we get the data set from Radish.sourceforg.net. This data set has recorded measurements from various sensors mounted on a mobile robot while running about $3.5 \mathrm{~km}$ for 30 mins in a underground mine. The measurement devices include inertial navigation system (INS), laser range finder and odometer, where INS is used to measure the range and angle of artificial landmark in the lane and laser range finder 
to get range $\mathrm{r}$ and orientation angle $\theta$. The sample period of laser range finder is $214 \mathrm{~ms}$ and the effective range is $30 \mathrm{~m}$ with $\theta$ in $(-\pi, \pi)$.

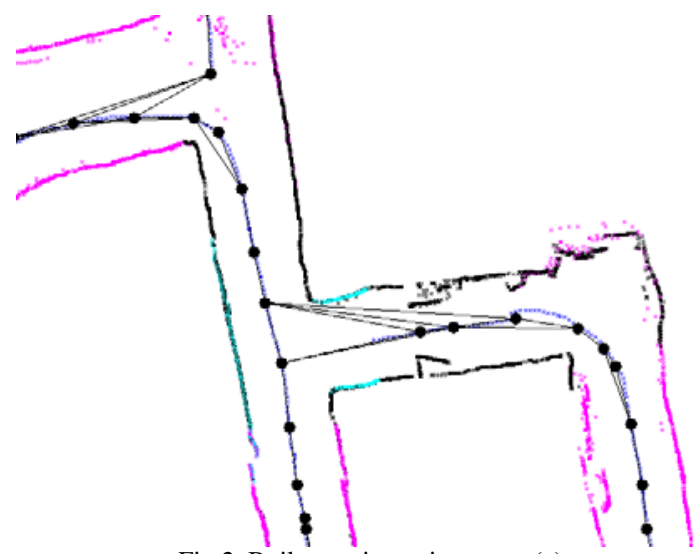

Fig 2. Built map in environment (a)

The NDT-EKF SLAM algorithm described in this paper has been extensively tested on the data set. Fig. 2 and Fig. 3 show the built maps in two cases. In Fig. 2, environment (a) has many branches and environment (b) has a big loop. In both cases, the NDT-EKF SLAM successfully built the maps. NDT-EKF SLAM algorithm has better localization precision. The reason for the relatively large error at start is due to the lack of environment information. The uncertainty in pose estimation diminishes as the number of scan matching and fusing process increases, which make the pose estimation to approximately reach real value.

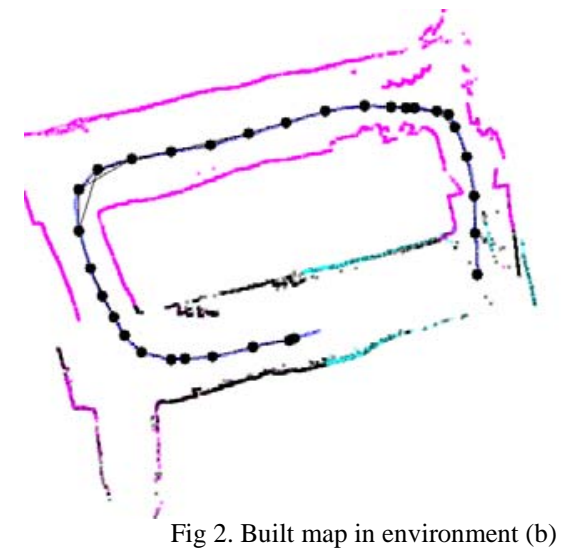

\section{CONCLUSION}

This paper studied SLAM for a rescue robot. It is difficult to extract typical features such as lines and planes in nonstructural rescue environment. Scan matching method based on normal distribution transform (NDT) can avoid the hard feature extraction problem by estimation of the probability distribution of laser scan data and location can be achieved using correlation of the NDT. Based on NDT scan matching, NDT-EKF SLAM algorithm has been proposed, which realizes fast and precise mapping for rescue environment by fusing odometry data and scan matching pose. The NDT-EKF SLAM algorithm described in this paper has been extensively tested and experimental results show that NDT-EKF SLAM algorithm is much better than other proposed SLAM algorithm.

\section{ACKNOWLEDGMENT}

This research is sponsored by Young Scientist Research Awards of Shandong Province(grant No. 2012BSB01383) and Research Fund for the Doctoral Program of Higher Education of China (grant No. 2010371820004), which are gratefully acknowledged.

\section{REFERENCES}

[1] K. Pathak, A. Birk, N. Vaskevicius. Online three-dimensional SLAM by registration of large planar surface segments and closed-form pose-graph relaxation. Journal of Field Robotics, vol. 27, 2010, pp. 52-84

[2] R. Murphy . Rescue robotics for homeland security. Communications of the ACM, special issue on homeland security, vol. 27, 2004, pp. 66-69

[3] S. Thrun, W. Thayer , C. Whittaker. Autonomous exploration and mapping of abandoned mines. IEEE Robotics and Automation Magazine, vol. 11, 2005, pp. 13-28

[4] P. Newman, G. Sibley, M. Cummins. Navigating, recognizing and describing urban spaces with vision and lasers. International Journal of Robotics Research, vol. 28, 2009, pp. 1406-1433

[5] R. Smith, M. Self, P. Cheeseman. Estimating uncertain spatial relationships in Robotics. Autonomous Vehicles. 1990, pp. 67-193

[6] S. Thrun, W. Burgard, D. Fox. Probabilistic Robotics. Cambridge, MA: the MIT press, 2005

[7] R.M. Eustice, H. Singh, J.J. Leonard. Exactly sparse delayed sate filters for view-based SLAM. IEEE Transaction on Robotics. vol. 22, 2006, pp. 1100-1114

[8] J. Nieto, T. Bailey, E. Nebot. Recursive scan-matching SLAM. Robotics and Autonomous Systems. vol. 55, 2007, pp. 39-49

[9] Nuchter, K. Lingermann, J. Hertzberg. 6D SLAM for 3D mapping outdoor environment. Journal of Field Robotics. vol. 24, 2007, pp. 242-249

[10] P.J. Besl, N.D. McKay. A method for registration of 3D shaps. IEEE Transaction on Pattern Analysis and Machine Intelligence. vol. 14, 1992, pp. 239-256

[11] F. Lu, E. Millios. Robot pose estimation in unknown environment by matching 2d range scans. Journal of Intelligent Robotics Systems, vol. 18, 1997, pp. 249-257

[12] P. Biber. The normal distribution transform: a new approach to laser scan matching. Proc. IEEE/RSJ(IROS’03), 2003, pp. 2743-2748 